\title{
Comparative study of the performance of semiconductor laser based coherent Doppler
} lidars

Rodrigo, Peter John; Pedersen, Christian

\section{Published in:}

Proceedings of SPIE

Link to article, DOI:

$10.1117 / 12.908800$

Publication date:

2012

Document Version

Publisher's PDF, also known as Version of record

Link back to DTU Orbit

Citation (APA):

Rodrigo, P. J., \& Pedersen, C. (2012). Comparative study of the performance of semiconductor laser based coherent Doppler lidars. In Proceedings of SPIE (Vol. 8241). [824112] SPIE - International Society for Optical Engineering. Proceedings of SPIE - The International Society for Optical Engineering https://doi.org/10.1117/12.908800

\section{General rights}

Copyright and moral rights for the publications made accessible in the public portal are retained by the authors and/or other copyright owners and it is a condition of accessing publications that users recognise and abide by the legal requirements associated with these rights.

- Users may download and print one copy of any publication from the public portal for the purpose of private study or research.

- You may not further distribute the material or use it for any profit-making activity or commercial gain

- You may freely distribute the URL identifying the publication in the public portal 


\section{Comparative study of the performance of semiconductor laser based coherent Doppler lidars}

Rodrigo, Peter John, Pedersen, Christian

Peter John Rodrigo, Christian Pedersen, "Comparative study of the performance of semiconductor laser based coherent Doppler lidars," Proc. SPIE 8241, High-Power Diode Laser Technology and Applications X, 824112 (8 February 2012); doi: 10.1117/12.908800

SPIE. Event: SPIE LASE, 2012, San Francisco, California, United States 


\title{
Comparative study of the performance of semiconductor laser based coherent Doppler Lidars
}

\author{
Peter John Rodrigo* and Christian Pedersen \\ DTU Fotonik, Department of Photonics Engineering, Technical University of Denmark, 4000 \\ Roskilde, Denmark
}

\begin{abstract}
Coherent Doppler Lidars (CDLs), operating at an eye-safe 1.5-micron wavelength, have found promising applications in the optimization of wind-power production. To meet the wind-energy sector's impending demand for more cost-efficient industrial sensors, we have focused on the development of continuous-wave CDL systems using compact, inexpensive semiconductor laser (SL) sources. As a preliminary part of our work, we compare the performance of two candidate emitters for an all-semiconductor CDL system: (1) a monolithic master-oscillator-power-amplifier (MOPA) SL and (2) an external-cavity tapered diode laser (ECTDL).
\end{abstract}

Keywords: Semiconductor laser, LIDAR

\section{INTRODUCTION}

In the wind-energy industry, velocity-measuring coherent Doppler Lidar (CDL) operating at the telecom wavelength $\lambda \sim 1.5 \mu \mathrm{m}$ is becoming a popular alternative to conventional anemometry instruments (i.e. fixed sensors like wind cups, wind vanes and sonic-anemometers) [1-3]. Furthermore, new studies have shown that wind turbines with control systems relying on remote wind velocity measurements of forward-looking, nacelle-mounted CDLs produce $10-20 \%$ higher power-production than a conventionally controlled turbine [4-5]. In order to scale-up such substantial improvement in wind harvest, low-cost, compact, mass-producible CDLs need to be developed. One promising approach is to employ semiconductor laser (SL) sources in CDL systems.

In 2000, researchers who initially considered SL-based CDLs identified several problems associated with the use of diode laser sources - e.g. low output power, phase-induced intensity noise (PIIN) [6-7]. Due to these challenges, Lidar developers redirected their attention to embodiments based on a more expensive laser technology, namely, the fiber-laser and fiber-amplifier (FLFA) tandem. Recently, we initiated a reinvestigation of SL-based CDL systems to tackle the abovementioned problems [8-10]. Our efforts focused on two novel SL sources as potential emitters for developing an "all-semiconductor-laser" wind Lidar for field operation.

In this work, we demonstrate that two SL sources, namely (1) a 1550-nm monolithic master-oscillator-power-amplifier (MOPA) SL and (2) a 1470-nm external-cavity tapered diode laser (ECTDL), have great potential in competing with expensive FLFA sources used in today's commercial CDLs. In the following section, we show that the MOPA-SL and the ECTDL are capable of achieving high output power up to $0.6 \mathrm{~W}$ and $0.5 \mathrm{~W}$, respectively - the power levels required in most wind Lidar applications. We also show in section 3 the measured spectral characteristics of both SL sources depicting single-frequency operation and high side-mode suppression ratio (SMSR $>40 \mathrm{~dB}$ ), i.e. performance at par with that of a Fiber Laser. In section 4, we present our measurements that quantitatively compare the relative intensity noise (RIN) properties of the MOPA-SL and the ECTDL versus that of a FL. Among the two SL sources, the monolithic MOPA-SL is found to be more reliable and even better in terms of RIN characteristics when compared to the FL. Furthermore, we present in section 5 the delayed self-homodyne measurement of the MOPA-SL's linewidth to deduce the potential range capability of a CDL based on this light source. Finally, in section 6 , we summarize the comparative study performed in this work and discuss some future extensions we plan to undertake.

*E-mail: pejr@fotonik.dtu.dk

High-Power Diode Laser Technology and Applications X, edited by Mark S. Zediker, Proc. of SPIE Vol. 8241, 824112 · (c) 2012 SPIE · CCC code: 0277-786X/12/\$18 · doi: 10.1117/12.908800

Proc. of SPIE Vol. $8241824112-1$ 


\section{OUTPUT POWER LEVELS}

For most applications of Lidar systems in wind-energy research, high output power from a single-frequency laser is normally required. This is due to the fact that a CDL wind sensor relies on detecting a very low-power backscattered optical field (approximately $10^{-12}-10^{-11}$ of the Lidar transmit beam power) by analyzing the beat signal it creates upon coherent mixing with a sub-milliwatt reference beam, the intensity noise of which is typically the most dominant noise term that competes with the interferometric signal. Frequency (Fourier) analysis of the beat signal enables us to determine the Doppler shift caused by moving aerosols in the lower part of our atmospheric boundary layer. From the central Doppler shift frequency, $\Delta f_{\mathrm{c}}$, the proportional wind speed component, $v_{\mathrm{r}}$, along the transmit beam is easily deduced using $v_{\mathrm{r}}=\lambda \Delta f_{\mathrm{c}} / 2$.

To be operational in various locations that cover a broad range of backscattering coefficients, commercial cw CDLs employ high power narrow-linewidth FLFA sources emitting watt-level average output power [1]. The most promising alternative to FLFA but employs an inexpensive "all-semiconductor" technology is found in SL emitters based on tapered semiconductor gain structures. In this work, two types of tapered SL sources are investigated: (1) 1550-nm MOPA-SL and (2) 1470-nm ECDTL.

The MOPA-SL used in this work is based on chip-level integration of a DFB semiconductor laser (MO section) and a tapered semiconductor amplifier (PA section). It comes in a hermetically sealed butterfly package with 1-meter polarization-maintaining (PM) fiber pigtail. Due to fiber-coupling, spatially single-mode Gaussian beam is emitted out of the FC/APC connectorized end of the fiber. The light output power versus current $(\mathrm{L}-\mathrm{I})$ curves of the MOPA-SL are shown in Fig. 1. For this power measurement, a current of $500 \mathrm{~mA}$ for the $\mathrm{MO}$ section is used while the laser package is kept at $\mathrm{T}=20.0^{\circ} \mathrm{C}$. Note that an almost linear relationship is observed (red squares) between the laser power measured by a built-in photodiode and the applied amplifier current. This is indicative of an SL behavior in which the PA section nearly acts as a purely passive amplifier for the field emitted from the adjacent laser cavity defined by the MO section, even at high amplifier current. It has been shown that the degree of astigmatism of the beam emitted from the PA front facet varies by hundreds of micrometers with drive current to the tapered section [11]. For this reason the coupling optics in the butterfly package are fixed in positions that optimize coupling efficiency at high operating current. Thus, at lower amplifier current, the resulting coupling efficiency is suboptimal, which explains the nonlinear profile of the measured power at the fiber exit versus current (blue diamonds).

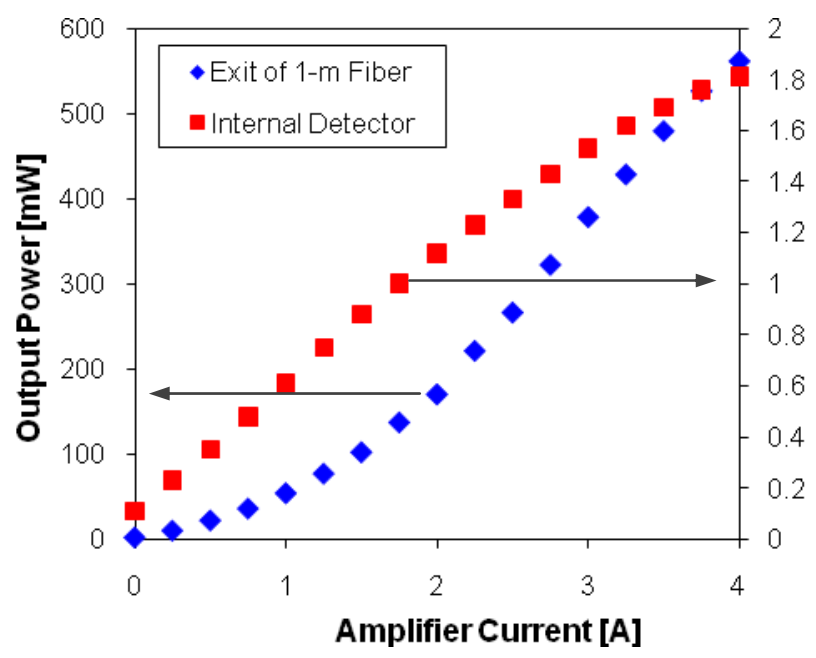

Figure 1. L-I characteristics of a 1550-nm MOPA-SL in a butterfly package with a PM-fiber pigtail. The output power measured at the tip of the fiber pigtail (diamonds) exceeds $0.5 \mathrm{~W}$ at $4.0 \mathrm{~A}$ of drive current to the PA section of the laser (drive current to the MO section is $500 \mathrm{~mA} ; \mathrm{T}=20.0{ }^{\circ} \mathrm{C}$ ). The nearly constant slope of the internally measured relative power of the MOPA versus PA current (squares) is not reproduced in the former L-I curve because the astigmatism of the front facet output beam coupled to the fiber depends on drive current and thus influences coupling efficiency. 
The ECTDL studied here was constructed from a ridge-waveguide tapered semiconductor device (InGaAsP/InP) with anti-reflection coatings on both facets. The performance of such tapered diode in an external cavity setup has already been demonstrated [12]. However, instead of using a ruled grating we have employed a volume Bragg grating (VBG) as the frequency selective element for the external cavity. The use of VBG allows us to adopt the focused beam geometry employed by G. Lucas-Leclin, et al. for 810-nm system, which was found to be more mechanically robust than using collimated beam in external cavity [13]. The reflective VBG (Ondax, 114-er149-001) used has a specified Bragg wavelength, diffraction efficiency and bandwidth of $1472 \pm 1 \mathrm{~nm}, 15 \%$ and $0.5 \mathrm{~nm}$, respectively. It is placed $25 \mathrm{~mm}$ from the rear facet of the ridge section or the plane on which the rear beam gets focused by an aspheric lens $(\mathrm{f}=4.5$ $\mathrm{mm})$. The front facet output beam is collimated by the combination of a second aspheric lens $(\mathrm{f}=4.5 \mathrm{~mm})$ and a cylindrical lens ( $\sim 50 \mathrm{~mm}$ ). The L - I curve of our ECTDL in Fig. 2 (blue diamonds) shows the measured power after the cylindrical lens as a function of drive current at $\mathrm{T}=20.0^{\circ} \mathrm{C}$ when the $\mathrm{VBG}$ is optimally aligned at the rear facet. The threshold current of $\sim 1.7 \mathrm{~A}$ and the observed slope efficiency are reminiscent of those in Ref. 12. The measured power of the amplified spontaneous emission (ASE) when the VBG is removed is also shown (red squares). Higher output power may be achievable using custom VBG with higher diffraction efficiency.

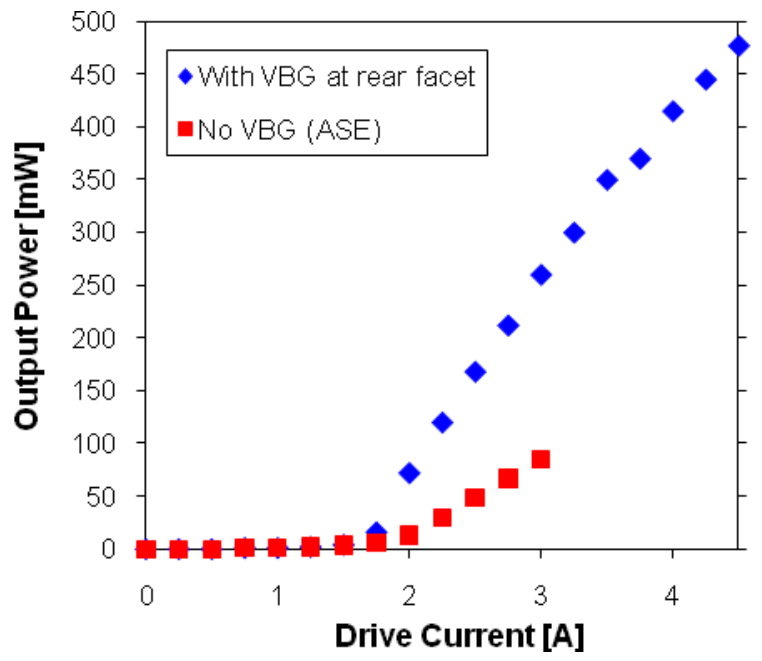

Figure 2. L-I characteristics of a 1470-nm ECTDL. Front facet output power measured after a collimation optics (diamonds) can reach $\sim 0.5 \mathrm{~W}$ at $4.5 \mathrm{~A}$ of drive current $\left(\mathrm{T}=20.0{ }^{\circ} \mathrm{C}\right)$. The spectrally selective feedback element used in this laser is a volume Bragg grating (VBG) placed $2.5 \mathrm{~mm}$ behind the rear facet of the tapered semiconductor gain medium. The measured front facet power of the gain medium when the VBG is removed (i.e. amplified spontaneous emission or ASE) is also plotted (squares).

\section{SPECTRAL CHARACTERISTICS}

To explore the differences in the performance of the MOPA-SL, the ECTDL and the most common CDL source - a FL (Koheras, Adjustik $\lambda=1575 \mathrm{~nm}$ ), we performed a number of measurements characterizing their spectral signatures using an optical spectrum analyzer (OSA). The OSA (Ando, AQ6315E) used in these measurements, set in doublemonochromator mode, has a resolution limited to $\sim 50 \mathrm{pm}$. The results are shown in Fig. 3. In Fig. 3a we can see that the measured linewidth of the FL is limited by the OSA resolution. In addition, the SMSR is very high $\geq 60 \mathrm{~dB}-$ the actual side-mode discrimination is possibly limited by the noise floor (dynamic range) of the OSA. For the MOPA-SL we obtained the laser spectra for four different amplifier current settings $(1.5 \mathrm{~A}-4.0 \mathrm{~A})$ at $\mathrm{MO}$ current of $500 \mathrm{~mA}$ and laser temperature $\mathrm{T}=20.0{ }^{\circ} \mathrm{C}$ (see Fig. 3b). Also high SMSR values around $55 \mathrm{~dB}$ are observed for the MOPA-SL with the center wavelength varying from $1548.9 \mathrm{~nm}$ to $1549.1 \mathrm{~nm}$ with increasing amplifier current. On the other hand, the laser line of the ECTDL at $\mathrm{T}=20.0^{\circ} \mathrm{C}$ appears locked at the Bragg wavelength of $1472.5 \mathrm{~nm}$ and stable over the range of drive current $(1.7 \mathrm{~A}-3.5 \mathrm{~A})$ with a deviation below $0.2 \mathrm{~nm}$ (Fig. 3c). The ECTDL mode-suppression for abovethreshold drive current, however, is lower than for the other two lasers (SMSR $\sim 40 \mathrm{~dB}$ ). In Fig. 4d, we further compared 
the spectral measurements of the OSA for the $14 \mathrm{xx} \mathrm{nm}$ tapered diode at 2.0-A drive current with and without the external-cavity grating. We note that the lasing line defined by the VBG is relatively different from the peak wavelength $(\sim 1460 \mathrm{~nm})$ of the tapered diode gain. This is one factor for the lower side-mode discrimination of our current ECTDL.
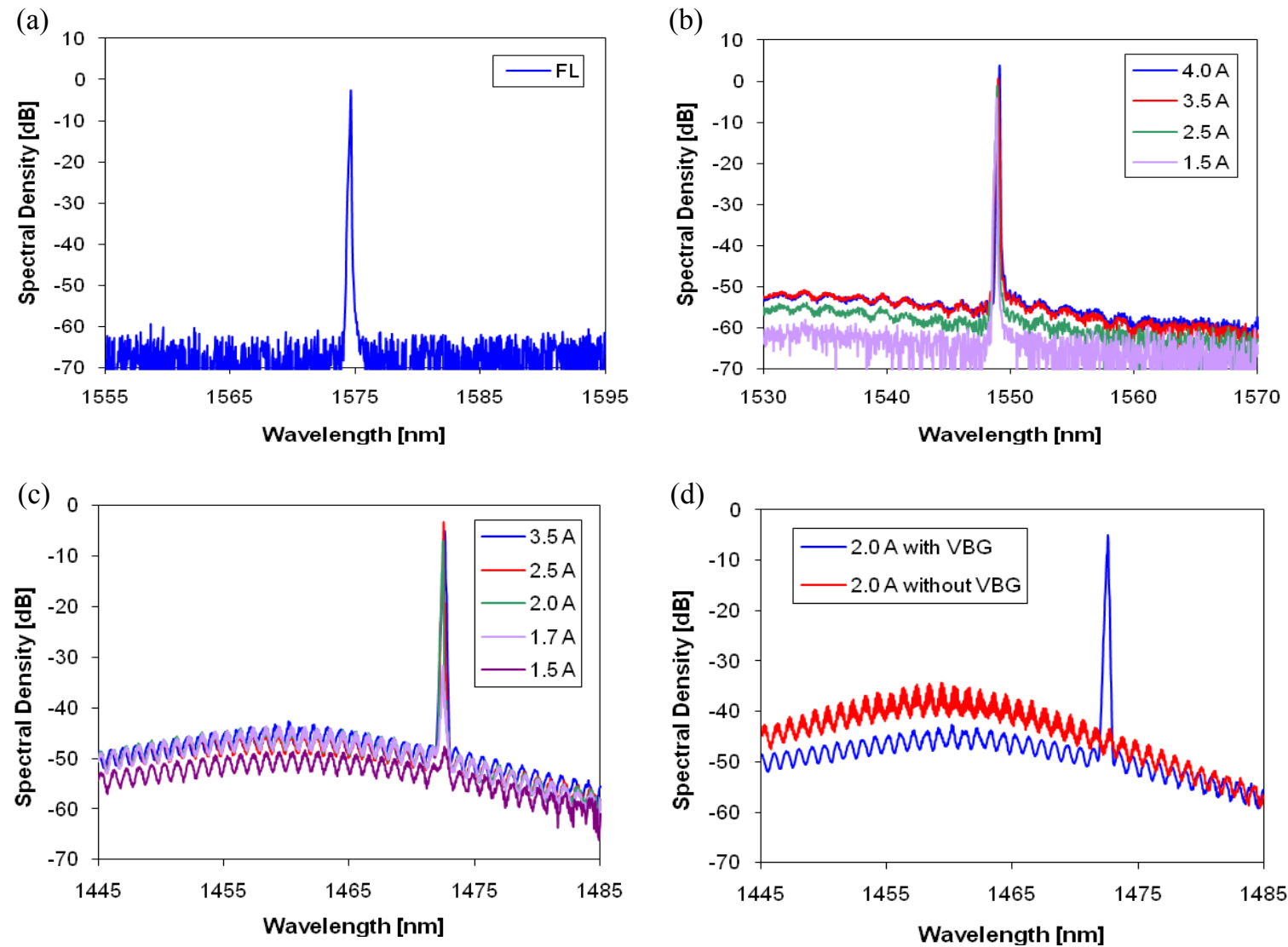

Figure 3. OSA-measured profiles of the laser emission of (a) a 1575-nm FL ( $\left.\mathrm{T}=25.0{ }^{\circ} \mathrm{C}\right)$, (b) a monolithic $1550-\mathrm{nm}$ MOPA-SL, and (c) a 1472-nm ECTDL using a VBG. (d) Re-plot of the profile of the ECTDL at 2.0 A drive current (blue curve) along with the resulting spectrum (ASE, red curve) when the VBG is blocked.

\section{RELATIVE INTENSITY NOISE}

Aside from high output power requirement and single-frequency operation, it is also important that the laser source of a CDL system possesses the lowest possible intensity noise over the relevant frequency bandwidth where the Doppler spectrum of the wind is expected to appear. For wind-turbine applications, this would be the range associated with LOS wind speeds $0<v_{\mathrm{r}}<30 \mathrm{~ms}^{-1}$ or $0<\Delta f_{\mathrm{c}}<40 \mathrm{MHz}$. It is thus worthwhile to quantitatively compare the intensity noise characteristics of the 1550-nm MOPA-SL and the 1470-nm ECTDL to that of the FL. To perform this comparative test, we used a fast ac-coupled InGaAs photoreceiver with built-in electronic amplifier. The photoreceiver output voltage (proportional to photocurrent) is sent to a data acquisition system which allows us to obtain and analyze the power density of the intensity noise for each laser under test. The responsivity of the InGaAs detector from $1470 \mathrm{~nm}$ to 1575 is relatively constant so that a side-by-side RIN comparison can be made by sending the same amount of power $(\sim 0.375$ $\mathrm{mW}$ ) from each laser to the active area of the detector. Figure 3 shows the RIN measurements for the three lasers as well as the detector unit noise floor. Our empirical results clearly indicate the superior RIN characteristics of the monolithic MOPA-SL over that of the present ECTDL. Moreover, the MOPA-SL exhibits a near-shot-noise-limited RIN profile over the entire frequency range considered relevant for wind sensing. This is not the case for the FL profile due to its known relaxation oscillation peak below $1 \mathrm{MHz}$, which lifts the noise floor from $3 \mathrm{~dB}$ to tens of $\mathrm{dB}$ above shot-noise 
level at frequencies below $\sim 7 \mathrm{MHz}$. Moreover, the effective noise floor of an FL when combined with a high-gain EDFA is further increased depending on the fiber amplifier's noise figure - lifting the noise level by at least $3 \mathrm{~dB}$ [14]. Thus, we expect that the SNR (i.e. data availability) for a CDL based on the MOPA-SL is potentially higher than that of an FLbased CDL particularly during low wind speed conditions. On the other hand, the observed intensity noise profile for our 1470-nm ECTDL is much higher than for the MOPA-SL. Furthermore, the ECTDL noise floor is unstable in terms of average level and profile, which can appear modulated over the frequency range. We also observed that even when a beam block is inserted between the VBG and the rear facet, the detected ASE exhibits intensity noise $10 \mathrm{~dB}$ above the shot-noise level though the average incident power is much lower. Nonetheless, this may be related to the observed increase in the magnitude of the ASE profile when the VBG is blocked as measured by the OSA (see Fig. 3d).

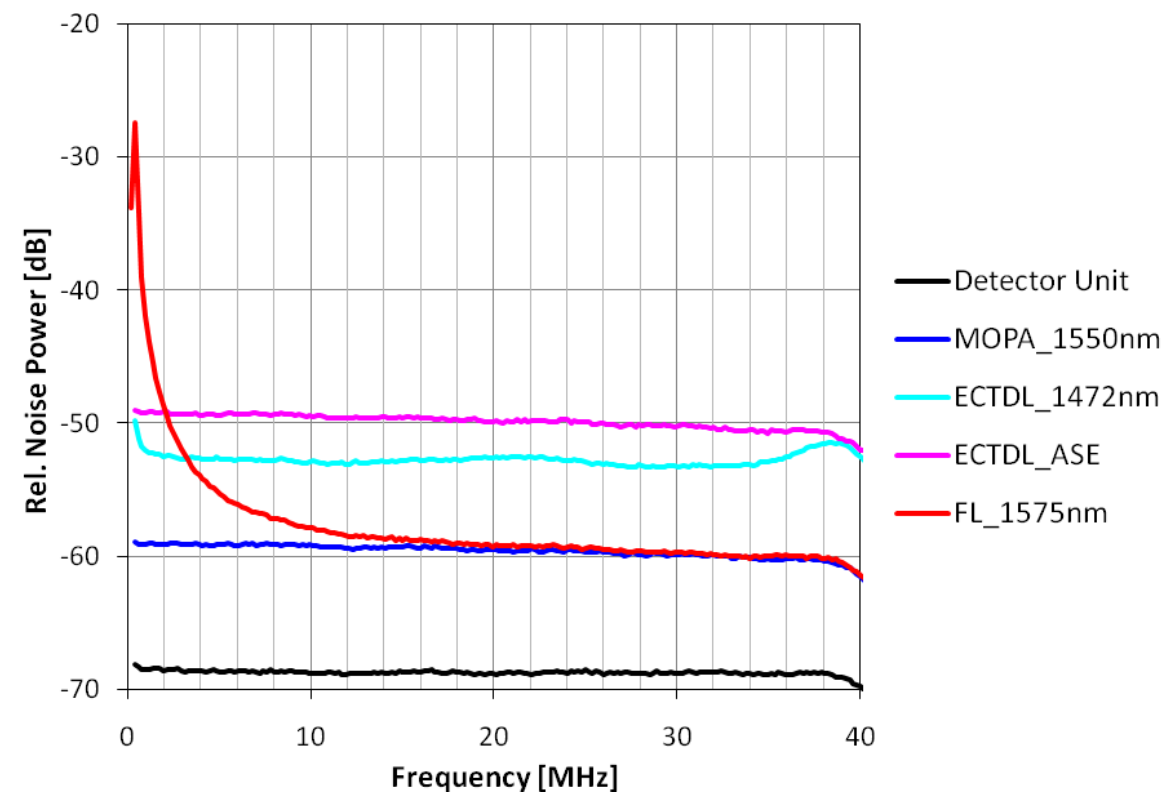

Figure 4. Comparison of the power densities of the intensity noise of the 1550-nm MOPA-SL, 1470-nm ECTDL and a 1575-nm fiber laser (FL) relative to the noise floor of the detector unit. The output power from each laser incident to the active area of the detector was $0.375 \mathrm{~mW}$. The spectrum of the ASE noise produced when the VBG of the ECTDL is removed is also plotted.

\section{MOPA-SL LINEWIDTH MEASUREMENT}

The highly stable shot-noise limited intensity noise property of the MOPA-SL allowed us to probe the laser's linewidth using an all-fiber self-homodyne setup shown in Fig. 5. In our self-homodyne setup, the output beam from the fibercoupled MOPA-SL is first sent through a fiber-optic isolator (FOI) with $\sim 40 \mathrm{~dB}$ isolation to protect the SL from unwanted optical feedback. Then the laser emission is split using a 90/10 fiber-splitter. A 4-km fiber is attached to the splitter arm with lower transmission and the other end of this fiber delay line is connected to the higher transmission port of a second 90/10 splitter used as a combiner. The remaining splitter and combiner ports are connected to form the undelayed arm of the interferometer. The output port of the 90/10 combiner is attached to a manually adjustable fiberoptic attenuator (FOA) so that the strength of the homodyne field incident to the photoreceiver can be controlled (e.g. to avoid detector saturation). The self-homodyne measurement data is plotted in Fig. 6. For a differential time delay of $19.6 \mu \mathrm{s}$, we find the data fits a Lorentzian profile with full-width at half-maximum (FWHM) of approximately $320 \mathrm{kHz}$, which suggests a MOPA-SL linewidth of about $160 \mathrm{kHz}$. This is comparable to other experimental data found in the literature $[8,15]$. Such linewidth corresponds to a suitable few hundred meters of coherence length - the typical remote sensing range required in most wind-energy applications of CDL. However, the measured MOPA-SL linewidth is several orders of magnitude larger than that of a FL, which means that PIIN (phase-related noise) may introduce deleterious excess noise if not mitigated $[6-7,10]$. 


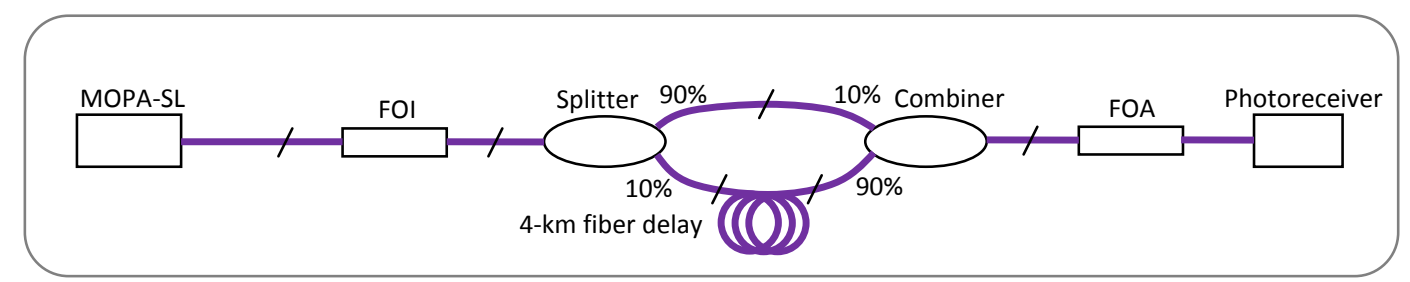

Figure 5. Self-homodyne setup for MOPA-SL linewidth determination. A 4-km delay corresponding to a differential time of $\sim 20 \mu \mathrm{s}$ is used. FOI, fiber-optic isolator; FOA, fiber-optic attenuator. The slanted line denotes FC/APC connections.

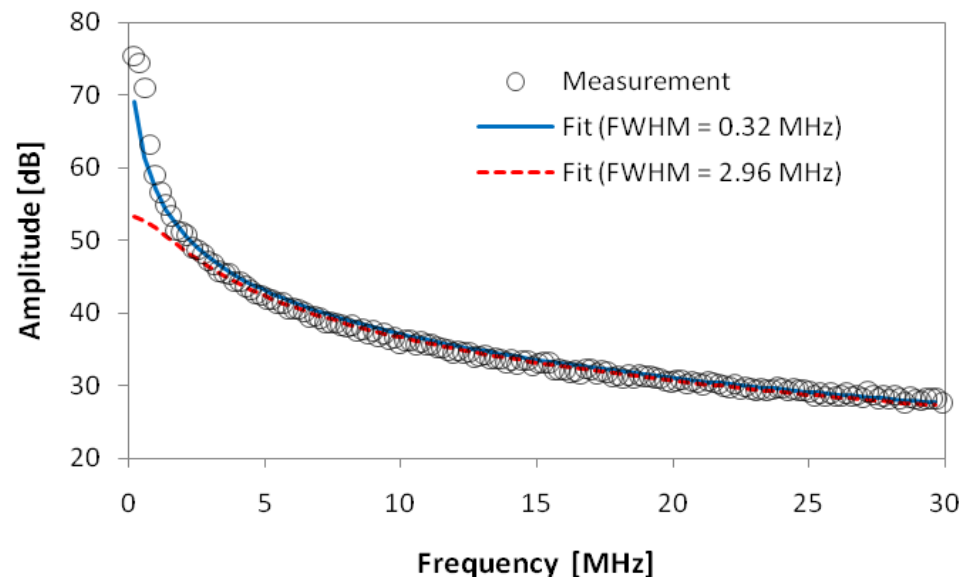

Figure 6. Self-homodyne linewidth measurement for the laser emission of the monolithic MOPA-SL operating at MO current of $500 \mathrm{~mA}$, PA current of $3.93 \mathrm{~A}$ and $\mathrm{T}=20.0^{\circ} \mathrm{C}$. The experimental data (circles) is in good agreement with a Lorentzian curve fit (solid line) corresponding to a laser linewidth of $160 \mathrm{kHz}$. A Lorentzian fit (dashed line) for a linewidth of $1.48 \mathrm{MHz}$ is also plotted for comparison.

\section{SUMMARY AND OUTLOOK}

A comparative investigation of novel high-power narrow-linewidth semiconductor lasers for CDL applications was presented in this work. Two SL systems were considered: (1) a 1550-nm MOPA-SL and (2) a 1472-nm ECTDL with a VBG. Both emitters were shown here to provide output power levels reaching $0.5 \mathrm{~W}$, which is comparable to the power level of FLFA systems currently employed in a number of commercially sold Lidar wind sensors. OSA measurements revealed the different center wavelengths of the two SLs and showed that the ECTDL's laser line is more robust to drive current (and also temperature) variation than for the MOPA. However, the OSA spectra showed higher SMSR values for the MOPA-SL almost similar to those observed in a FL $(\sim 60 \mathrm{~dB})$. The superiority of the MOPA-SL over the ECTDL is further observed in the intensity noise characterization. Aside from the absence of a sub-MHz relaxation oscillation peak inherently present in the FL intensity noise, the shot-noise limited RIN floor of the MOPA-SL is also stable over the bandwidth relevant for wind sensing applications. This stable performance enabled us to measure the linewidth of the MOPA-SL using a homodyne setup, which we estimate to be about $160 \mathrm{kHz}$. These comparative results are summarized in Table 1 below along with additional parameters.

For future studies, we will try to improve the performance of the ECTDL emitter using better optimized VBG. Specifically, we believe that the slope efficiency and the side-mode suppression can be enhanced by using VBG with higher diffraction efficiency (note that the current VBG has $\sim 15 \%$ efficiency). Furthermore, the SMSR can also improve significantly if the grating is designed with a Bragg wavelength that closely coincides with the peak of tapered device gain. An improvement in the SMSR of the ECTDL is likely to translate into better RIN performance [16]. 
In the summer of 2011, we have deployed in the field our CDL system based on the fiber-coupled MOPA-SL studied in this work. The promising results of the first outdoor test of the MOPA-SL based CDL will be published in a separate report [17]. The next stages of development that we envision is to extend the capabilities of our unique all-semiconductor CDL system for simultaneous measurement of both wind speed and wind direction with high accuracy.

Table 1. Comparison of two semiconductor laser sources and a fiber laser for CDL application

\begin{tabular}{|l|l|l|l|}
\hline & MOPA-SL & ECTDL & Fiber Laser + EDFA \\
\hline Wavelength [nm] & 1550 & 1470 & $1535-1575$ \\
\hline Power [W] & 0.6 & 0.5 & $\geq 1.0$ \\
\hline RIN [dB above shot-noise] & $\sim 0 @ 1-100 \mathrm{MHz}$ & $\sim 10 @ 1-100 \mathrm{MHz}$ & $\sim 30 @<1 \mathrm{MHz} ; 3 @ 7$ \\
& & MHz; $\sim 0 @ 15-100 \mathrm{MHz}$ \\
\hline Linewidth [kHz] & $100-500$ (measured) & $10-50$ (expected) & $<1$ \\
\hline PIIN sensitivity & High & Moderate & Low \\
\hline Coherence length [km] & $0.2-1$ & $2-10$ & 100 \\
\hline Compactness & Very Compact & Compact & Bulky \\
\hline Mechanical Stability & High & Average & High \\
\hline
\end{tabular}

\section{REFERENCES}

[1] www.yourwindlidar.com

[2] www.catchthewindinc.com

[3] www.aventlidartechnology.com

[4] Catch the Wind, Inc., "Boosting Power Production," Dr. Elizabeth Dakin, December 2009.

[5] Catch the Wind, Inc., "Catching the Wind: An Update on Improved Yaw Alignment," Dr. Elizabeth Dakin, Dr. Priyavadan Mamidipudi and Dr. Andrew Hopkins, March 2011.

[6] M Harris, G. N. Pearson, J. M. Vaughan, D. Letalick, and C. J. Karlsson, "The role of laser coherence length in continuous-wave coherent laser radar," J. Mod. Opt. 45, 1567-1581 (1998).

[7] C. J. Karlsson, F. Å. A. Olsson, D. Letalick, and M. Harris, "All-fiber multifunction continuous-wave coherent laser radar at $1.55 \mu \mathrm{m}$ for range, speed, vibration, and wind measurements," Appl. Opt. 39, 3716-3726 (2000).

[8] R. S. Hansen and C. Pedersen, "All semiconductor laser Doppler anemometer at $1.55 \mu \mathrm{m}$," Opt. Express 16, 18288-18295 (2008).

[9] P. J. Rodrigo and C. Pedersen, "Doppler wind lidar using a MOPA semiconductor laser at stable singlefrequency operation," In: Technical Digest. 19th International Congress on Photonics in Europe, CLEO/EuropeEQEC 2009.

[10] P. J. Rodrigo and C. Pedersen, "Reduction of phase-induced intensity noise in a fiber-based coherent Doppler lidar using polarization control," Opt. Express 18, 5320-5327 (2010).

[11] S. O'Brien, D. F. Welch, R. A. Parke, D. Mehuys, K. Dzurko, R. J. Lang, R. Waarts, and D. Scifres, “Operating Characteristics of a High-Power Monolithically Integrated Flared Amplifier Master Oscillator Power Amplifier,” IEEE J. Quantum Electron. 29(6), 2052-2057 (1993).

[12] S. Kallenbach, M. T. Kelemen, R. Aidam, R. Lösch, G. Kaufel, M. Mikulla, and G. Weimann, "High-Power High-Brightness Ridge-Waveguide Tapered Diode Lasers at 14xx nm," Proc. SPIE 5738, 406-415 (2005).

[13] G. Lucas-Leclin, D. Paboeuf, P. Georges, J. Holm, P. Andersen, B. Sumpf, and G. Erbert, "Wavelength stabilization of extended-cavity tapered lasers with volume Bragg gratings," Appl. Phys. B 91, 493-498 (2008).

[14] www.rp-photonics.com/erbium doped fiber amplifiers.html

[15] W. Liang, N. Satyan, A. Yariv, A. Kewitsch, G. Rakuljic, F. Aflatouni, H. Hashemi, and J. Ungar, "Coherent power combination of two Master-oscillator-power-amplifier (MOPA) semiconductor lasers using optical phase lock loops," Opt. Express 15, 3201-3205 (2007).

[16] C. B. Su, J. Schlafer, and R. B. Lauer, "Explanation of low-frequency relative intensity noise in semiconductor lasers," Appl. Phys. Lett. 57, 849-851 (1990).

[17] P. J. Rodrigo and C. Pedersen, to be submitted. 